\title{
Capsule commentary on Zhang et al., Race/ethnicity, Disability, and Medication Adherence Among Medicare Beneficiaries with Heart Failure
}

\author{
Paul A. Heidenreich, MD, MS \\ Veterans Administration Palo Alto Healthcare System, 111 C Cardiology, Palo Alto, CA, USA.
}

J Gen Intern Med 29(4):648

DOI: $10.1007 / \mathrm{s} 11606-013-2736-2$

(c) Society of General Internal Medicine 2014

I $\mathrm{n}$ an analysis of Medicare patients with heart failure, Zhang and Baik found strong racial differences in adherence to medical treatment. ${ }^{1}$ Native American, black, and Hispanic patients were the least likely to be adherent to common heart failure medications [beta-blockers, angiotensin converting enzyme (ACE) inhibitors, or diuretics], followed by Asian and then white patients. The same pattern was seen for disabled patients, with adherence rates lower for all groups. While white patients were the most adherent, the absolute values were still concerning as only $63 \%$ were adherent (defined as having enough medications to cover $75 \%$ of days). Left ventricular ejection fraction (LVEF) data were not available; among those with a high LVEF, discontinuation of these medications would not be expected to impact survival. Moreover, regardless of the LVEF, the need for diuretics may wax and wane. However, even though some of the discontinuation may be appropriate, it is highly likely that much of the non-adherence occurred at the patient's discretion and may involve lack of adherence with medications known to prolong survival. The low rate for black patients is of particular concern since prior studies of hospitalized patients have found that they are more likely to have a lower LVEF than whites ${ }^{2,3}$ and thus are more likely to benefit from therapy.

There are several implications for the practicing provider. The authors recommend providers be particularly sensitive to medication use by disabled and non-white patients. However, attention to barriers to medication use for all patients is needed given the magnitude of non-adherence seen for all groups. Medication reviews are time-consuming and difficult to complete during a typical clinic visit. Fortunately, several electronic medical record systems can now provide up-to-date medication possession ratios for each of a patient's medications. One can envision future automated systems where a letter is sent or other contact made with a potentially non-adherent patient to determine barriers to care. As the authors note, reducing medication costs will not eliminate the adherence problem. In many cases an individualized intervention will be needed that considers medical knowledge, attitudes toward medical care and preferences for length versus quality of life.

Conflict of Interest: The author declares no conflicts with this article.

Corresponding Author: Paul A. Heidenreich, MD, MS; Veterans Administration Palo Alto Healthcare System, 111C Cardiology, 3801 Miranda Avenue, Palo Alto, CA 94306, USA (e-mail: heiden@stanford.edu).

\section{REFERENCES}

1. Zhang Y, Baik SH. Race/ethnicity, disability, and medication adherence among Medicare beneficiaries with heart failure. J Gen Intern Med. 2013; doi: 10.1007/s11606-013-2692-x.

2. Kamath SA, Drazner MH, Wynne J, Fonarow GC, Yancy CW. Characteristics and outcomes in African American patients with decompensated heart failure. Arch Intern Med. 2008;168(11):11521158.

3. Echols MR, Felker GM, Thomas KL, Pieper KS, Garg J, Cuffe MS, Gheorghiade M, Califf RM, O'Connor CM. Racial differences in the characteristics of patients admitted for acute decompensated heart failure and their relation to outcomes: results from the OPTIME-CHF trial. J Card Fail. 2006;12(9):684-688. 